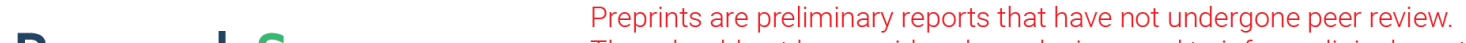 They should not be considered conclusive, used to inform clinical practice, or referenced by the media as validated information. \\ Fast Determination of the Rubber Content in Taraxacum Kok-Saghyz Fresh Biomass Using Portable Near Infrared Spectroscopy and Pyrolysis- Gas Chromatography
}

\section{Ying Chen}

Beijing University of Chemical Technology

Dong Yiyang ( $\nabla$ yydong@mail.buct.edu.cn)

Beijing University of Chemical Technology https://orcid.org/0000-0003-1752-3935

\section{Xiang Ma}

Beijing University of Chemical Technology

Jiaru Li

Beijing University of Chemical Technology

\section{Minmin Guo}

Beijing University of Chemical Technology

Jichuan Zhang

Beijing University of Chemical Technology

\section{Research}

Keywords: Taraxacum kok-saghyz, Rubber, Near infrared spectroscopy, Pyrolysis gas chromatography

Posted Date: March 16th, 2021

DOI: https://doi.org/10.21203/rs.3.rs-298105/v1

License: (c) (i) This work is licensed under a Creative Commons Attribution 4.0 International License.

Read Full License

Version of Record: A version of this preprint was published at Journal of Analysis and Testing on March 29th, 2022. See the published version at https://doi.org/10.1007/s41664-022-00217-z. 


\section{Abstract}

Background: Taraxacum kok-saghyz (TKS), a plant native to the Tianshan valley on the border between China and Kazakhstan and inherently rich in natural rubber, inulin and other bioactive ingredients, is an important industrial crop. TKS rubber is a good substitute for natural rubber. TKS's breeding work necessitates the need to screen high-yielding varieties, hence rapid determination of rubber content is essential for the screening. Conventional analytical methods cannot meet actual needs in terms of realtime testing and economic cost. Near-infrared spectroscopy analysis technology, which has developed rapidly in the field of industrial process analysis in recent years, is a green detection technology with obvious merits of fast measurement speed, low cost and no sample loss. This research aims to develop a portable non-destructive near-infrared spectroscopic detection scheme to evaluate the content of natural rubber in TKS fresh roots. Pyrolysis gas chromatography (PyGC), was chosen as the reference method for the development of NIR prediction model.

Results: 208 TKS fresh root samples were collected from the Inner Mongolia Autonomous Region of China. Near-infrared spectra were acquired for all samples. Randomly two-thirds of them were selected as the calibration set, the remaining one-third as the verification set, and the partial least squares method was successfully used to establish a good NIR prediction model at $1080-1800 \mathrm{~nm}$ with a performance to deviation ratio (RPD) of 5.54 and coefficient of determination $\left(R^{2}\right)$ of 0.95 .

Conclusions: This study showed that portable near-infrared spectroscopy could be used with ease for large-scale screening of TKS plants in farmland, and could greatly facilitate TKS germplasm preservation, high-yield cultivation, environment-friendly, high-efficiency and low-cost rubber extraction, and comprehensive advancement of the dandelion rubber industry thereof.

\section{Background}

Natural rubber (NR) is a vital commodity in world market due to its excellent physical properties that synthetic rubber cannot replicate or prevail. It can be used to manufacture more than 40,000 consumer products. In the past decade, the world's demand for natural rubber has increased dramatically. Alternative sources of natural rubber will not only increase the supply of rubber, but will also provide resource protection for countries engaged in planting. More than 2500 kinds of plants can synthesize natural rubber, most of which produce small quantities of low molecular weight rubber. Only Hevea brasiliensis or para rubber tree, Parathenium argentatum and Taraxacum kok-saghyz can produce rubber with molecular weight over $1000 \mathrm{kDa}$ and have in turn applicative values (1). Hevea brasiliensis is currently the only large-scale commercialized rubber-producing plant, mainly distributed in tropical and subtropical regions such as Asia, Africa and South America. Asian rubber production accounts for $93 \%$ of the world's rubber production, while the output from South America as the origin of the Hevea brasiliensis which is easily affected by leaf blight, only accounts for $c a .2 \%$. As a single rubber-producing plant, Hevea brasiliensis may face potential threats. Countries have gradually realized the importance of developing 
natural rubber resources other than Para rubber, and have successively initiated the research and development of Hevea brasiliensis alternative rubber-producing crops (2).

Taraxacum kok-saghyz (TKS), is a rubber-producing plant native to the mountains of Kazakhstan and the Tianshan Valley-Tex River Basin in Xinjiang, China. TKS plant, inherently rich in natural rubber, inulin and other bioactive ingredients e.g. penta-cyclic triterpenoids, is an important industrial crop. Discovered in the 1930's by the former U.S.S.R exploration team (3), TKS was further investigated to reduce dependence on foreign rubber sources. The mass fraction of rubber within TKS's taproot can reach $2.89 \%-27.89 \%$ (3), and TKS rubber is of good quality, with a molecular weight of more than $100,0000 \mathrm{~g} / \mathrm{mol}$ (4). In addition, gloves and other rubber products made of traditional rubber may cause personal allergic reaction, namely life-threatening Type $\otimes H e v-b$ latex allergy, while the rubber of TKS has better biocompatibility and can be used to manufacture hyposensitive medical goods (5). In addition, TKS is easy to grow, which has strong ability to grow in both cold areas and temperate regions, and to resist bacterial infections and pest invasions.

The determination of rubber content is crucial for the growth of alternative rubber plants in the natural rubber industry. The breeding of TKS necessitates the screening of high-yielding varieties, thus rapid determination of rubber content is essential for the work. Over the years, a variety of techniques have been used to determine rubber content, such as gravimetry (6), gel permeation chromatography (GPC) (7), infrared spectroscopy (IRS) (8), mass spectrometry (MS) $(9,10)$, etc. However, quantification is usually a time-consuming process, which slows down the speed of improving rubber quality and yield through breeding. Several quantitative procedures have been discussed to measure the rubber content in plants $(11,12)$. In most cases the protocols comprise two successive steps, extraction of rubber, then quantification of the components by gravimetry, chromatography, or a spectral technique (13-19). For example, quantitative Soxhlet extraction method was used as the main technique for rubber quantitation (20). With this method, plant materials were first ground into small particles, and the dry-milled materials were put into cellulose extraction thimbles. Acetone extraction for 4 hours was next used to remove resins, and then samples were extracted with hexane for another 4 hours to obtain the rubber. The collected extractants were evaporated, leaving dry film of rubber. Following evaporation of the solvents, weights were determined for rubber contents using gravimetric analysis (11). This method requires timeconsuming purification and extraction steps, and consumes a lot of organic solvents. Moreover, the rubber is easily degraded in boiling acetone, which affects the quality of the rubber consequently.

Pyrolysis gas chromatography (PyGC) has been used to determine the rubber content of plant-derived natural rubber e.g., Hevea brasiliensis, Eucommia ulmoides and synthetic rubber (21). The principle of pyrolysis gas chromatography is easy to understand. Firstly, the sample is heated in a strictly controlled environment. During the heating process, the target polymeric compound is thermally and quantitatively cracked to small volatile molecule compounds, then these mingled small molecules are separated by gas chromatography to obtain a chromatogram. Finally, the polymeric structure and composition of the sample can be inferred based on the qualitative and quantitative data analysis (8). Pyrolysis gas chromatography is a relatively accurate method so far to determine the rubber content indirectly. The 
method is fast and accurate which does not require complicated sample processing and rubber extraction steps, and eliminates the need of any organic reagents. The rubber content data of a plant sample can be easily acquired in an average of 40 minutes, and both dry and wet samples can be measured.

Near infrared spectroscopy (NIRS) is based on an electromagnetic radiation wave between visible (Vis) and mid-infrared (MIR). The near-infrared region, defined as 780-2526nm by the American Society of Testing Materials (ASTM), is the first invisible region found in the absorption spectrum. The near infrared spectrum originates from the combinatoriallly vibrational/rotational frequency and doubling absorption frequency regions of hydrogen groups $(\mathrm{OH}, \mathrm{NH}, \mathrm{CH})$ in organic molecules. By scanning with ease, the near infrared spectra of the samples, the characteristic fruitful information of hydrogen groups in organic molecules can be obtained. Sample analysis by near infrared spectroscopy is deemed to be convenient, rapid, efficient, accurate, cost effective, non-destructive and environmentally friend. Therefore, this technology is favored by more and more natural rubber researchers (22).

The main purpose of this study is to develop a facile near-infrared spectroscopic method to determine the content of rubber in the fresh root of Taraxacum kok-saghyz. By collecting different varieties of TKS samples and taking the measured values of pyrolysis gas chromatography as the reference data of the NIR model, the quantitative prediction model of rubber content was established by multivariate regression analysis method. The prediction accuracy and reproducibility of the analysis model were evaluated, and a set of near infrared spectrum model suitable for the fresh root of TKS was explored.

\section{Material And Methods}

\subsection{Biomass samples}

The samples used in this study were collected from the TKS plantation base in Duolun County, Inner Mongolia Autonomous Region, China. A total of 50 fresh TKS with entire roots of different varieties and different growth stages were collected in 2019 and 2020.

\subsection{Experimental protocol}

In order to obtain clean rubber roots for spectroscopic analysis, the picked and collected TKS roots were washed to remove external impurities and sludge. The washed fresh roots were wiped with tissue paper and weighed to get the mass of fresh TKS before dehydration. In order to reduce the error of moisture fluctuation, each plant sample needed to be weighed again before being irradiated with near-infrared spectrometer, to ensure that the mass difference incurred by the water loss was between $8 \%-10 \%$. Each TKS plant was categorized into main root and lateral roots, the main root and two to three lateral roots were selected at the definite position (main root: $10-20 \mathrm{~mm}$ under the leaf, lateral roots: $5-15 \mathrm{~mm}$ under the main root junction) to be irradiated for near infrared spectroscopy. NIR light-irradiated position of each plant was marked to facilitate successive determination of reference rubber content values by thermal pyrolysis gas chromatography. A total of 208 TKS root samples were collected. 


\subsection{Pyrolysis gas chromatography}

In this study, pyrolysis gas chromatography was used as the reference method to measure the rubber content of TKS for the development of NIR prediction model. The sample was pretreated by freeze-drying and grinding. The fresh TKS root samples with collected near infrared spectra were placed in a vacuum freeze dryer. After freeze-dried for 2 days, TKS root samples were ground into a uniform fine powder. Then, the ground samples were stored in sealed bags, labeled and placed in a desiccator.

Next, the pyrolysis gas chromatography measurement was performed. An electronic balance with an accuracy of $0.01 \mathrm{mg}$ was used to weigh and record the tare weight of the pyrolysis sample cup. Ca. 5-10 $\mathrm{mg}$ of the powdered samples were weighed and put into the sample cup. Then the mass before pyrolysis was weighed on the balance, and recorded. An Agilent $6890 \mathrm{~N}$ gas chromatography equipped with a PY UA- 5 capillary column $(30.0 \mathrm{~m} \times 250 \mu \mathrm{m} \times 0.25 \mu \mathrm{m})$ and a thermal conductivity detector $(T C D)$ was used for the experiment.

Thermal pyrolysis conditions: thermal pyrolysis temperature $550^{\circ} \mathrm{C}$, pyrolysis time $0.1 \mathrm{~s}$. Gas chromatographic conditions: injection port temperature $250^{\circ} \mathrm{C}$, split ratio $50: 1$, carrier gas was high-purity nitrogen, the initial temperature of the programmed column temperature was $40^{\circ} \mathrm{C}$, and the initial temperature was kept for 5 minutes, then the temperature was increased to $130^{\circ} \mathrm{C}$ at a rate of $10^{\circ} \mathrm{C} / \mathrm{min}$ and maintained for 10 minutes. Finally, the temperature was increased to $280^{\circ} \mathrm{C}$ at a rate of $10^{\circ} \mathrm{C} / \mathrm{min}$ and kept for 20 minutes.

When preparative work was done, warmup and condition the pyrolysis-gas chromatographic instrument, put the sample cup into the inlet of the facility. The gaseous pyrolyzed small molecule components of the sample were blown into the gas chromatograph with nitrogen through the pyrolysis injection needle, and then the chromatogram was obtained for the detection. The tared sample mass and the corresponding peak area of pyrolyzed limonene were substituted into our reported calibration curve (23) to obtain the reference value of TKS rubber content.

\subsection{Near infrared spectroscopy}

A self-assembled portable near-infrared spectrometer in the laboratory (Figure. 1) was used to collect the near-infrared reflectance spectra of TKS plant samples. The spectrometer consists of a halogen tungsten light source, grating, detector, and a reflective optical fiber. The optical resolution is $6.25 \mathrm{~nm}$, and data can be collected in the 900-2500 nm spectral range. In order to avoid direct contact, the reflective fiber probe was $5 \mathrm{~mm}$ away from the sample, which could keep the probe clean and protected the probe from scratches. In the following experiments, in order to reduce light scattering, a hand-held gun-like scanner was specially designed for the probe, and the probe was retracted $5 \mathrm{~mm}$ into the scanner head. Before each sample scan, a PTFE reference plate was utilized to determine the absolute reflectance baseline. To ensure measurement accuracy, the absolute reflectance reference was calibrated every 15 minutes during spectrum acquisition. 


\subsection{Statistical analysis}

Chemometrics software package Thermo GRAMS was used to develop rubber content prediction models, where GRAMS AI module was used to preprocess the spectrum, GRAMS IQ module was used to build the model, and IQ Predict module was used to predict and evaluate the model. The data matrix required to establish a rubber prediction model needed to correlate the standard rubber content value with the corresponding near-infrared spectrum. The Mean Center spectrum preprocessing method was used to subtract the average spectrum from each individual spectrum, which could eliminate data offset and make the characteristic difference between sample spectra more obvious. Standard Normal Variate Transformation (SNV) mathematically corrected the spectrum. This correction reduced the influence of the size of solid particles, surface scattering, and changes in optical path on the near-infrared spectrum. Then the second derivative with a smoothing range of 12 data points and a cubic polynomial was used to eliminate interference from the baseline and other backgrounds, distinguish overlapping peaks, and improve resolution and sensitivity (24). For fresh roots, the water band was removed at wavelength intervals of $1350-1450 \mathrm{~nm}$ and $1800-1950 \mathrm{~nm}$. A calibration equation was developed using partial least squares (PLS) regression method. For cross-validation, a set of NIRS measurements was divided into 25 subsets, which have randomly selected measurements. The calibration set included 140 measurements, and the validation set included 68 measurements (208 measurements in total).

\section{Results And Discussion}

\subsection{Analysis of the TKS samples by the reference method (PyGC)}

Each of the 208 samples was prepared in accordance with the procedure detailed in Sect. 2. The powder samples of TKS dry roots that had been ground for use were directly subjected to thermal pyrolysis gas chromatography determination. After thermal pyrolysis, the measured peak area of the pyrolyzed product at the designated time and the sample injection volume (mass) were substituted into the calibration curve equation $y=343269.93 x+1520710.36$ to calculate the rubber content of the sample. According to the ratio of the measured rubber content to the sample injection volume, the percentage or mass fraction of rubber content was obtained.

Table 1 showed the descriptive statistics of moisture and NR content after pyrolysis gas chromatography. The number of samples was 208. The moisture content was calculated based on wet biomass. The average moisture content of all samples was $71.58 \%$, and the standard deviation was $1.72 \%$. 
Table 1

Descriptive statistics of moisture and natural rubber contents after PyGC determination.

\begin{tabular}{|llllll|}
\hline Parameters & $\begin{array}{l}\text { Minimum } \\
\text { value }\end{array}$ & $\begin{array}{l}\text { Maximum } \\
\text { value }\end{array}$ & $\begin{array}{l}\text { Mean } \\
\text { value }\end{array}$ & $\begin{array}{l}\text { Standard } \\
\text { deviation }\end{array}$ & $\begin{array}{l}\text { Number of } \\
\text { samples }\end{array}$ \\
\hline Moisture content (\%) & 69.23 & 75.16 & 71.58 & 1.72 & 208 \\
$\begin{array}{l}\text { Natural rubber } \\
\text { content (\%) }\end{array}$ & 0.20 & 16.72 & 5.00 & 2.64 & \\
\cline { 1 - 3 } & & & & & \\
\hline
\end{tabular}

The rubber content of all samples ranged from $0.2-16.72 \%$, with an average value of $5.00 \%$ and a standard deviation of $2.64 \%$. Therefore, the analysis work showed that these 208 samples covered an ideal variation range of these extractable biomass components in agronomic and breeding research work (25).

\subsection{Near infrared spectroscopy predictive model of TKS rubber}

\subsubsection{Estimation of natural rubber content}

After preprocessing, the spectrum data was imported into Grams IQ chemometrics software together with the reference value. Randomized two-thirds of all samples were used as the calibration dataset, and the remaining one-third were used as the verification dataset. Partial Least Squares Regression (PLSR) was used to establish the relationship between spectral data and sample composition data. Using crossvalidation as a verification method, a single data pair (spectrum and analyte) was deleted from the dataset, and the remaining data was used to predict unknown composition values from the spectral data. This process was then repeated in the entire data set to test its prediction accuracy, so that the $\mathrm{R}^{2}$ of the model and the standard error of cross-validation could be calculated. The best model had the lowest standard error, and the $\mathrm{R}^{2}$ is close to 1 .

After the calibration model was established, the verification set was used to evaluate the accuracy and applicability of the model. IQ Predict was used to predict spectral data from the calibration model and compare it with the corresponding reference value to calculate the prediction standard deviation (SEP), the ratio of the verification set standard deviation to the prediction standard deviation (RPD), and the coefficient of determination $\left(R^{2}\right)$. These statistical parameters were used to evaluate the calibration model. The calculation formulas of SEP, RPD and $\mathrm{R}^{2}$ are as follows:

$$
S E P=\sqrt{\frac{\sum_{i=1}^{m}\left(y_{i, a c t u a l}-y_{i, p r e d i c t e d}\right)^{2}}{m-1}}
$$

where $\mathrm{y}_{i, \text { actual }}$ are the measured values of the reference method of the $i$-th sample; $y_{i, \text { predicted }}$ are the predicted values of the spectroscopic method of the $i$-th sample in the validation set prediction process; $m$ is the number of samples in the validation set. 


$$
\mathrm{RPD}=\frac{S D_{V}}{S E P}
$$

In the formula, $\mathrm{SD}_{\mathrm{v}}$ is the standard deviation of the concentration values of all samples in the validation set.

$$
R^{2}=1-\frac{\sum_{i=1}^{n}\left(y_{i, \text { actual }}-y_{i, p r e d i c t e d}\right)^{2}}{\sum_{i=1}^{n}\left(y_{i, \text { actual }}-\overline{y_{\text {actual }}}\right)^{2}}
$$

In the formula, $\mathrm{y}_{i, \text { actual }}$ is the measured value of the reference method of the $i$-th sample; $\overline{y_{\text {actual }}}$ is the average value of the measured value of the reference method of all samples in the calibration set or validation set; $\mathrm{y}_{i, \text { predicted }}$ is the predicted value of the $i$-th sample in the prediction process of the correction set or the verification set; $n$ is the number of samples in the correction set or the verification set.

The evaluation criteria are as follows: The smaller the SEP, the more accurate the result. Under the premise that the standard deviation (SDV) of the verification set is the same, the larger the $\mathrm{R}^{2}$, the higher the accuracy. Under the premise of the same concentration range, the larger the RPD, the higher the accuracy (24).

In this study, the Gap 2nd + SNV pretreatment method was used to develop the partial least squares (PLS) rubber prediction model. The total sample size was 90 where 4 abnormal data points were eliminated, two-thirds of all samples were selected as the calibration set, and one-third as the verification set. Table 2 showed the statistical properties of the NIRS model used to estimate rubber content. The cross-validation and verification results were similar. In the same way, the verification result was similar to the calibration result. Cross-validation and validation were related to the performance of the model used to estimate a single value. The $\mathrm{R}^{2}$ of the obtained model was 0.95 , and the standard error of cross validation (SECV) was 0.47 . The validation set data was used to evaluate the near-infrared rubber model, and the prediction software IQ Predict was used to make predictions. The standard error of prediction (SEP) of rubber was calculated to be 0.42 by predicting the rubber content and the known reference value. The SEP of the rubber model was close to the calibration SECV, indicating that the model was more accurate in predicting the verification set. Through calculation, it was verified that the $\mathrm{R}^{2}$ value was 0.95 , indicating that the model had the ability to predict rubber content. The ratio of performance to deviation (RPD) of the model was calculated to be 5.54. According to the evaluation standard, the prediction of the model was more accurate and could be used for quantification. Figure 2 showed the model of the NR obtained. 
Table 2

Descriptive statistics of natural rubber contents predicted by NIRS.

\begin{tabular}{|lllllll|}
\hline Constituent & $\mathbf{N}$ & Mean & $\mathbf{S D}_{\text {cal }}$ & SEC & $\mathbf{R}_{\mathbf{c}}{ }^{2}$ & RPD $_{\mathbf{c}}$ \\
\hline Calibration & 140 & 4.99 & 2.60 & 0.47 & 0.95 & 5.54 \\
Constituent & $\mathrm{N}$ & Mean & $\mathrm{SD}_{\text {val }}$ & $\mathrm{SEP}$ & $\mathrm{R}_{\mathrm{p}}{ }^{2}$ & $\mathrm{RPD}_{\mathrm{p}}$ \\
\hline Validation & 68 & 5.36 & 2.71 & 0.42 & 0.90 & 6.44 \\
\hline
\end{tabular}

Fresh roots contain a large proportion of water, and as the time of exposure to the environment increases, the water loss in the roots will also increase. Therefore, to ensure the robustness of the experiment, the influence of moisture content on the near-infrared spectrum must be minimized. In order to reduce the error of moisture detection, the surface soil of each plant was washed with clean water immediately after being picked, wiped dry and weighed, and weighed again before collecting near-infrared spectroscopy to ensure that the mass difference was maintained at ca. $8 \%$ because of the water loss. Thus, the characteristic peaks (at $1400 \mathrm{~nm}, 1900 \mathrm{~nm}$ ) of the obtained spectrum of water content were very small and could be ignored.

Another point that was prone to errors in the collection of fresh roots NIR spectra was the influence of the irradiation position. As shown in Fig. 3, it is not difficult to see the distribution of the laticifers after the root of TKS is cross-cut. The laticifers of TKS are arranged closely between the epidermis and xylem along a ring (26), and the laticifer cells in the laticifers are where the latex is produced. Since the laticifers are more evenly distributed in the roots, so long as each plant was irradiated at the definite position (main root: $10-20 \mathrm{~mm}$ under the leaf, lateral roots: $5-15 \mathrm{~mm}$ under the main root junction) to acquire near infrared spectrum, the error could be greatly reduced.

\subsubsection{Interpretation of NIRS spectra}

From the raw spectral data, it can be roughly seen that the absorption peaks associated with rubber are at 1300-1500 nm, 1600-1800 nm and 2100-2300 nm (Fig. 4). In order to extract the spectral characteristics, the data was processed using a preprocessing method to obtain clearer absorption peaks at $1350-1450 \mathrm{~nm}, 1650-1800 \mathrm{~nm}$ and $2150-2300 \mathrm{~nm}$ (Fig. 5). These bands are in keeping with observations done by (20) and (15). Since the compound absorption region of the near-infrared spectroscopy has many overlapping areas, various treatment methods such as derivative spectra were used to enhance the absorption peaks of $\mathrm{CH}, \mathrm{CH}_{2}$, and $\mathrm{CH}_{3}$ functional groups in isoprene to establish the correlation of the natural rubber concentration and the spectral absorption value. There are also rubber absorption peaks in the range of $2200-2400 \mathrm{~nm}$, but the spectral noise in this region is relatively strong. 


\subsection{Prediction by NIRS of rubber content values of fresh biomass}

IQ Predict was used to predict the spectral data from the calibration model and compare it with the corresponding reference value. 20 unknown TKS fresh root samples were predicted using the NIR model. The result was tabulated in Table 3. 
Table 3

Comparison of NIR model predicted value and reference value.

\begin{tabular}{|llll|}
\hline Samples & Reference value (\%) & Predicted value (\%) & Relative error (\%) \\
\hline 1 & 4.6 & 4.72 & 2.48 \\
\hline 2 & 9.41 & 10.15 & 7.32 \\
\hline 3 & 5.44 & 5.45 & 0.11 \\
\hline 4 & 4.74 & 5.06 & 6.37 \\
\hline 5 & 7.23 & 7.00 & -3.35 \\
\hline 6 & 5.13 & 5.67 & 9.48 \\
\hline 7 & 10.02 & 9.57 & -4.73 \\
\hline 8 & 13.02 & 13.04 & 0.13 \\
\hline 9 & 9.27 & 8.96 & -3.44 \\
\hline 10 & 4.83 & 4.93 & 1.97 \\
\hline 11 & 6.11 & 6.07 & -0.65 \\
\hline 12 & 7.02 & 6.69 & -4.87 \\
\hline 13 & 8.13 & 7.77 & -4.59 \\
\hline 14 & 6.2 & 5.71 & -8.55 \\
\hline 15 & 6.66 & 7.02 & 5.19 \\
\hline 16 & 9.43 & 9.04 & -4.28 \\
\hline 17 & 8.5 & 8.42 & -0.93 \\
\hline 18 & 4.43 & 4.92 & 10.04 \\
\hline 19 & 8.31 & 4.68 & -13 \\
\hline 20 & 4.18 & 56 & \\
\hline
\end{tabular}

It can be seen from the table that the relative errors between the predicted value of the model and the result determined by PyGC are almost within 10\%. The results in Table 3 show that the rubber content predicted by the NIR model is close to the rubber content measured using the reference method. It proves that the prediction results of this NIR model are relatively accurate and can be used for large-scale actual sample detection.

\section{Conclusion}

In our previous research, we washed and dried TKS fresh roots and pressed into tablets to collected nearinfrared spectra. This pre-processing method avoided the influence of moisture on near-infrared spectra. 
However, for the near-infrared spectroscopy detection method, its biggest advantage is fast and nondestructive, so the simpler the pre-processing work, the more it can reflect the advantages of the method in practical applications. More importantly, the simple pre-processing work can greatly reduce the human error in the operation process, which is extremely helpful to improve the accuracy of the near-infrared spectroscopy model. Therefore, in this study, we use straightforwardly fresh plant roots as the object of near-infrared spectroscopy. The research results $\left(R^{2}=0.95, R P D=5.54\right)$ are satisfactory and can meet the needs of practical applications.

The standard error of PyGC reference values used to calibrate the NIRS method is quite low at $2.64 \%$. In the characterization of macro or even trace amounts of natural rubber, pyrolysis gas chromatography is probably the most accurate method to date. In this study, the method was combined with near-infrared spectroscopy, and a reliable result with good consistency was obtained, which can accurately and quickly characterize the content of natural TKS rubber.

TKS is a crop with great potential. TKS contains not only natural rubber, but also raw materials such as inulin and protein that can be used for commercialization (27). The comprehensive development and utilization of TKS will be the development direction of this crop (28). In the future, near-infrared spectroscopy technology will also provide more convenience for realizing the comprehensive economic value of TKS.

In addition, although this spectral model for field measurement can be used to estimate the content for a single rubber plant, it is even more applicable for evaluating the average NR content in plants in the entire field to monitor the impact of planting parameters. For practical reasons, this research was mostly conducted in laboratory. The next step will be actual measurements on the ground to provide breeders, agronomists and farmers with a simple and high throughput tool for facile rubber content determinations.

\section{Declarations}

\section{Acknowledgments}

The authors are all grateful for the sponsorship provided by Beijing Linglong Dandelion Science \& Technology Development Co., Ltd.

\section{Authors' contributions}

YD and JZ supervised the conception and experiment; YC conducted the PyGC and NIR analyses; XM, JL and MG provided help on experimental operation; YC collected data and constructed PLSR models; YC wrote the manuscript; YD provided suggestions and comments for the manuscript; YC and YD revised the manuscript. All authors read and approved the manuscript.

\section{Funding}


This research was supported by the National Key Research and Development Program of China (Grant No. 2019YFF0302004).

\section{Availability of data and materials}

The datasets generated during and/or analyzed during the current study are available from the corresponding author on reasonable request.

\section{Ethics approval and consent to participate}

Not applicable.

\section{Consent for publication}

Not applicable.

\section{Competing interests}

The authors declare that they have no competing interests.

\section{References}

1. Mooibroek H, Cornish K. Alternative sources of natural rubber. Appl Microbiol Biotechnol. 2000;53:355-65.

2. Whalen M, McMahan C, Shintani D. Development of Crops to Produce Industrially Useful Natural Rubber. Isoprenoid Synthesis in Plants and Microorganisms2012. p. 329-45.

3. Krotkov G. A Review of Literature on Taraxacum Kok-Saghyz Rod. The Botanical Review. 1945;XI(8):417-61.

4. Zhang N, Guo T, Ma X, Liu J, Dong Y, Zhang J. Rational Rubber Extraction and Simultaneous Determination of Rubber Content and Molecular Weight Distribution in Taraxacum kok-saghyz Rodin by Size-Exclusion Chromatography. Chromatographia. 2019;82(10):1459-66.

5. Kutuzova SN, Brach NB, Kon'kova NG, Gavrilova VA. Taraxacum Kok-Saghyz (Asteraceae, Compositae) as A Source of Valuable Raw Materials for Rubber, Food, and Pharmaceutical Industries. Interdisciplinary Scientific Applied Journal. 2015;7(4):392-402.

6. Buranov AU, Elmuradov BJ. Extraction and characterization of latex and natural rubber from rubberbearing plants. J Agric Food Chem. 2010;58(2):734-43.

7. Guo T, Liu Y, Wei Y, Ma X, Fan Q, Ni J, et al. Simultaneous qualitation and quantitation of natural trans-1,4-polyisoprene from Eucommia ulmoides Oliver by gel permeation chromatography (GPC). J Chromatogr B Analyt Technol Biomed Life Sci. 2015;1004:17-22.

8. Takeno S, Bamba T, Nakazawa Y, Fukusaki E, Okazawa A, Kobayashi A. Quantification of trans-1,4polyisoprene in Eucommia ulmoides by fourier transform infrared spectroscopy and pyrolysis-gas chromatography/mass spectrometry. J Biosci Bioeng. 2008;105(4):355-9. 
9. Zhang X, Guo T, Xiang T, Dong Y, Zhang J, Zhang L. Quantitation of isoprenoids for natural rubber biosynthesis in natural rubber latex by liquid chromatography with tandem mass spectrometry. $J$ Chromatogr A. 2018;1558:115-9.

10. Xiang Tong GT, Zhang Xi C, Yunhan D, Yiyang Z, Jichuan M, Qiang ZL. Rapid Quantitative Determination of Isoprene Monomer in Living Taraxacum kok-saghyz by Ultra-High Performance Liquid Chromatography Tandem Mass Spectrometry. China Petroleum Processing Petrochemical Technology. 2020;22(2):30-6.

11. Black LT, Hamerstrand GE, Kwolek WF. Analysis of rubber, resin and moisture content of guayule by near infrared reflectance spectroscopy. Rubber ChemTechnol. 1985;58:304-13.

12. Wagner JPaP DG. Continuous Solvent Extraction Process for Recovery of Natural Rubber from Guayule. Polymer-Plastics Technology Engineering. 1988;27(3):335-50.

13. Takeno S, Bamba T, Nakazawa Y, Fukusaki E, Okazawa A, Kobayashi A. A high-throughput and solvent-free method for measurement of natural polyisoprene content in leaves by Fourier transform near infrared spectroscopy. J Biosci Bioeng. 2008;106(6):537-40.

14. Salvucci ME, Coffelt TA, Cornish K. Improved methods for extraction and quantification of resin and rubber from guayule. Ind Crops Prod. 2009;30(1):9-16.

15. Suchat S, Pioch D, Palu S, Tardan E, van Loo EN, Davrieux F. Fast determination of the resin and rubber content in Parthenium argentatum biomass using near infrared spectroscopy. Ind Crops Prod. 2013;45:44-51.

16. Ramirez-Cadavid DA, Valles-Ramirez S, Cornish K, Michel FC. Simultaneous quantification of rubber, inulin, and resins in Taraxacum kok-saghyz (TK) roots by sequential solvent extraction. Ind Crops Prod. 2018;122:647-56.

17. Luo Z, Thorp KR, Abdel-Haleem H. A high-throughput quantification of resin and rubber contents in Parthenium argentatum using near-infrared (NIR) spectroscopy. Plant Methods. 2019;15:154.

18. Ramirez-Cadavid DA, Cornish K, Hathwaik U, Kozak R, McMahan C, Michel FC. Development of novel processes for the aqueous extraction of natural rubber from Taraxacum kok-saghyz (TK). Journal of Chemical Technology Biotechnology. 2019;94(8):2452-64.

19. Taurines M, Brancheriau L, Palu S, Pioch D, Tardan E, Boutahar N, et al. Determination of natural rubber and resin content of guayule fresh biomass by near infrared spectroscopy. Ind Crops Prod. 2019;134:177-84.

20. Cornish K, Myers MD, Kelley SS. Latex quantification in homogenate and purified latex samples from various plant species using near infrared reflectance spectroscopy. Ind Crops Prod. 2004;19(3):28396.

21. Hu JC-A. Pyrolysis gas chromatography analysis of rubbers and other high polymers. Anal Chem. 1977;49(4):537-40.

22. Lu W. Modern Near Infrared Spectroscopy Analytical Technology (Second Edition). Beijing, China: China Petrochemical Press; 2006. 
23. Wang Lu GT, Zhang Qing M, Qiang D, Yiyang ZJ. Determination of Natural Rubber Content in Taraxacum Kok-Saghyz by Pyrolysis Gas Chromatography-Mass Spectrometry. China Petroleum Processing Petrochemical Technology. 2020;22(3):43-8.

24. Chu X. Molecular Spectroscopy Analytical Technology Combined with Chemometrics and its Applications. Beijing: Chemical Industry Press; 2011.

25. Arias $M$, Herrero J, Ricobaraza M, Hernández M, Ritter E. Evaluation of root biomass, rubber and inulincontents in nine Taraxacum koksaghyz Rodin populations. Ind Crops Prod. 2016;83:316-21.

26. Tobler CMaF. Zur Wissenschaftlichen und Praktischen Kenntnis der Kautschukpflanze Taraxacum Kok-Saghyz Rod. Planta. 1948;36:188-98.

27. Ramirez-Cadavid DA, Cornish K, Michel FC. Taraxacum kok-saghyz (TK): compositional analysis of a feedstock for natural rubber and other bioproducts. Ind Crops Prod. 2017;107:624-40.

28. Cornish K. Perspectives and Ongoing Challenges. The Rubber Tree Genome. Compendium of Plant Genomes2020. p. 169-75.

\section{Figures}




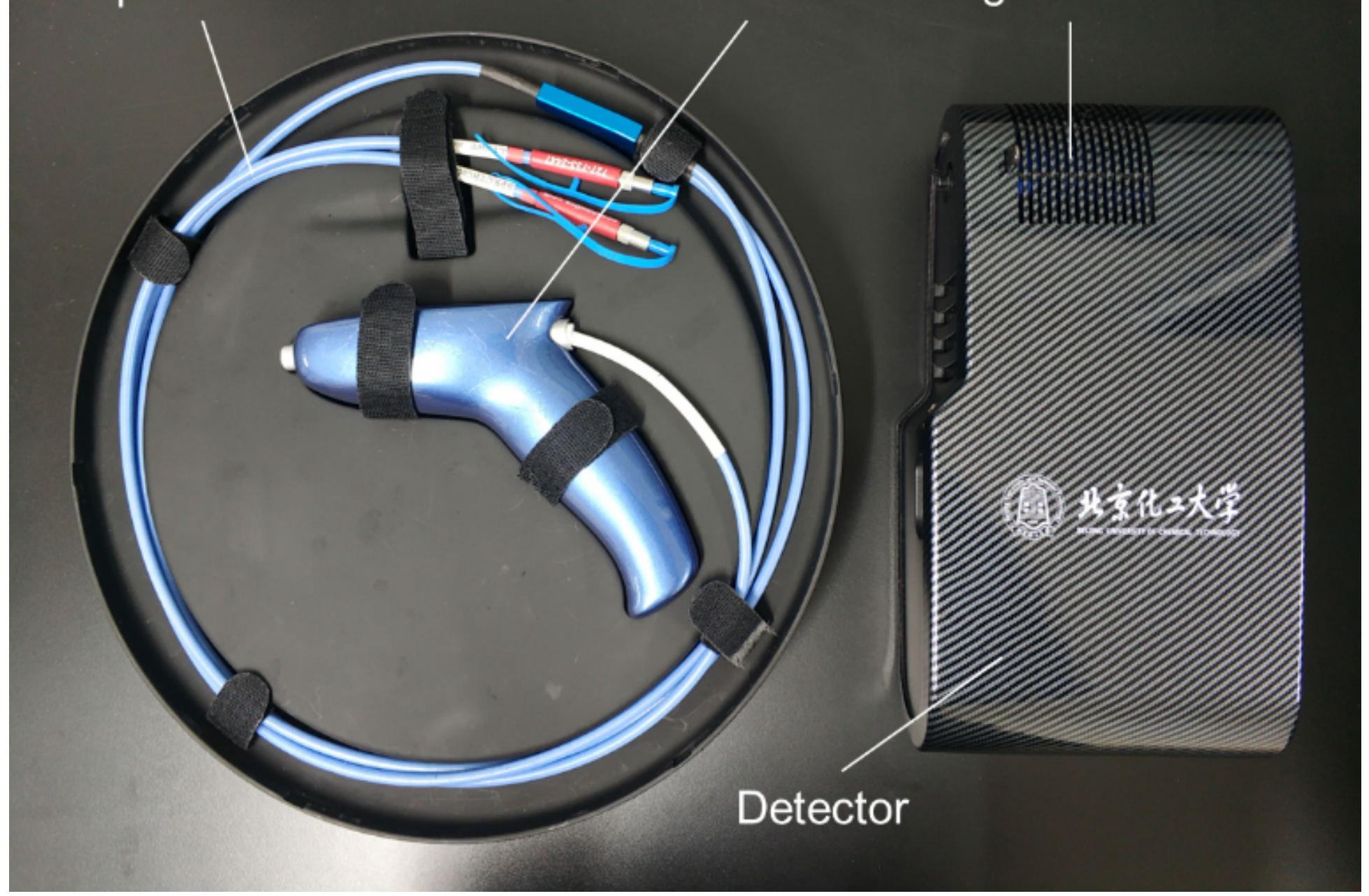

Figure 1

Laboratory self-assembled portable near-infrared spectrometer 


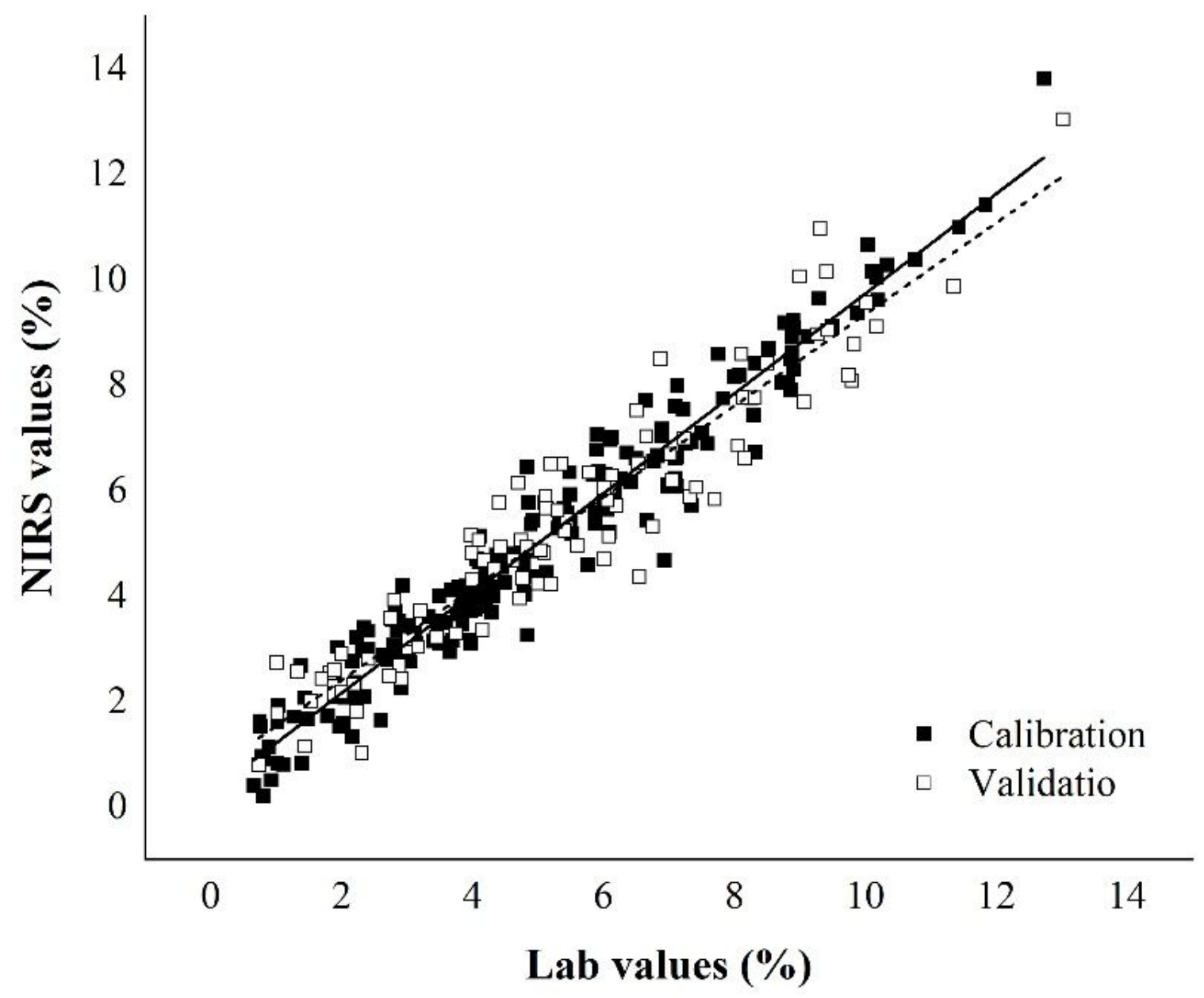

Figure 2

Scatter plot of measured and predicted values 


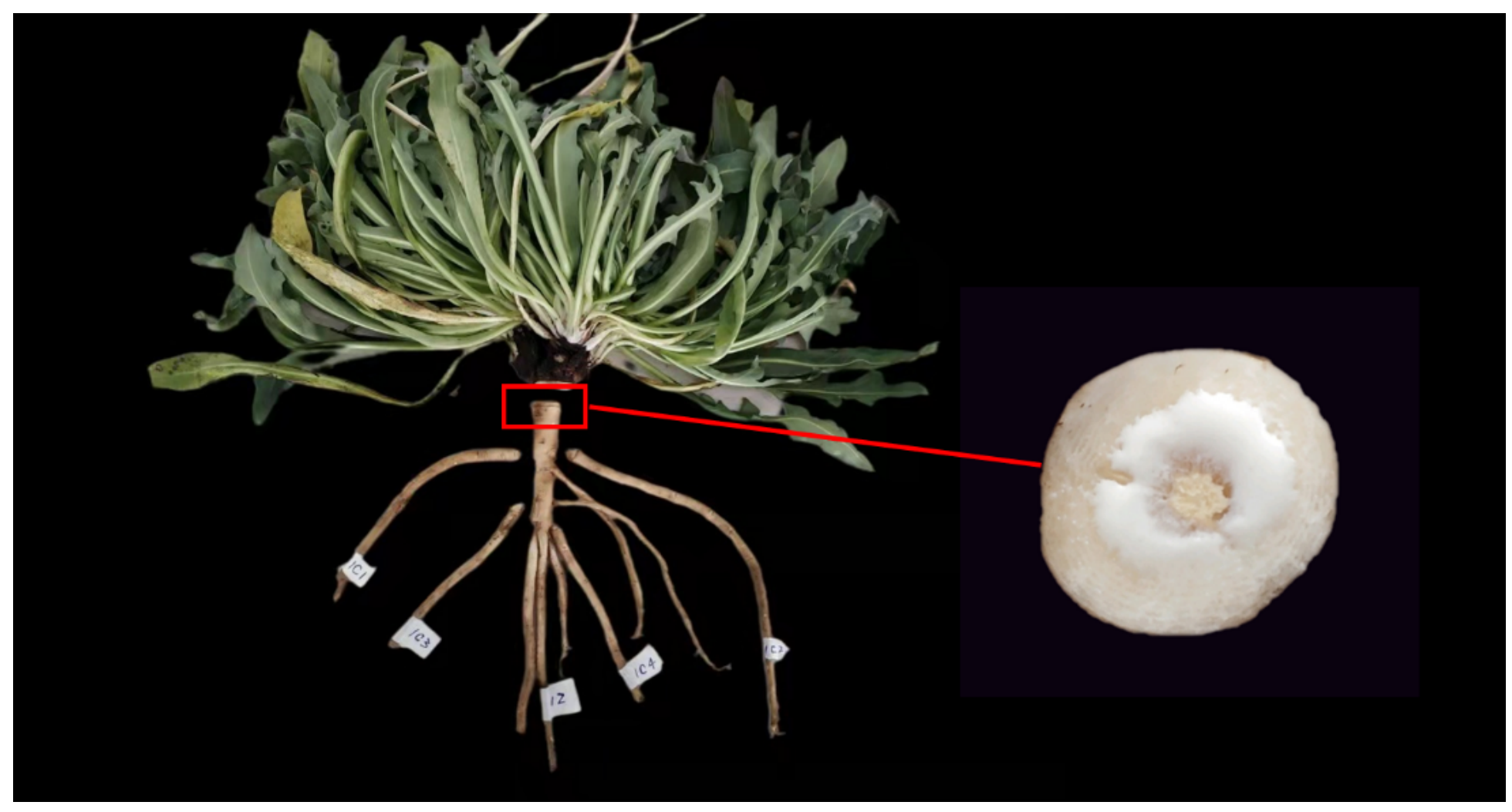

Figure 3

Fresh TKS and cross section with TKS latex secreted from laticifer cells

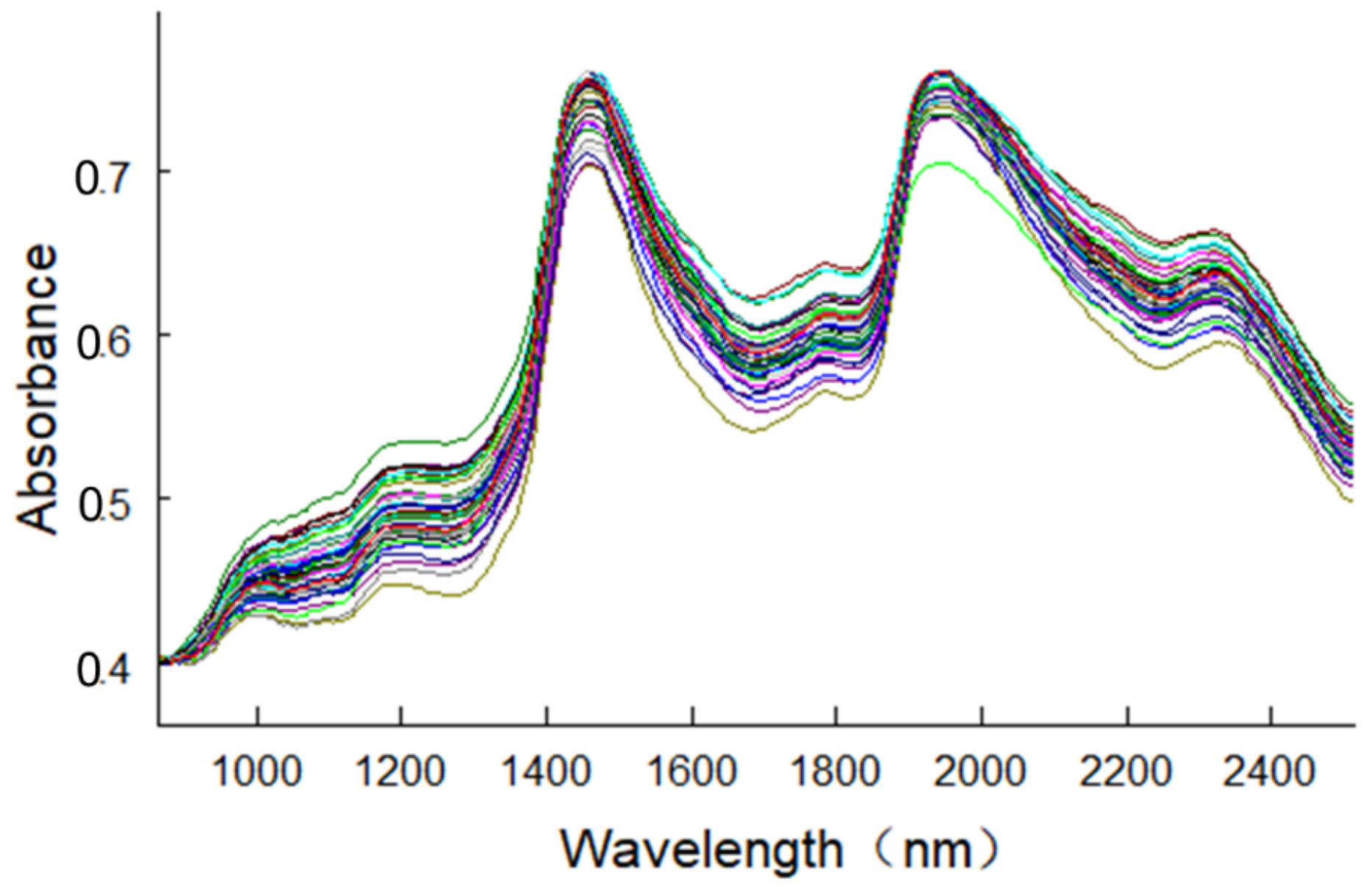


Figure 4

Raw near infrared spectra of the samples

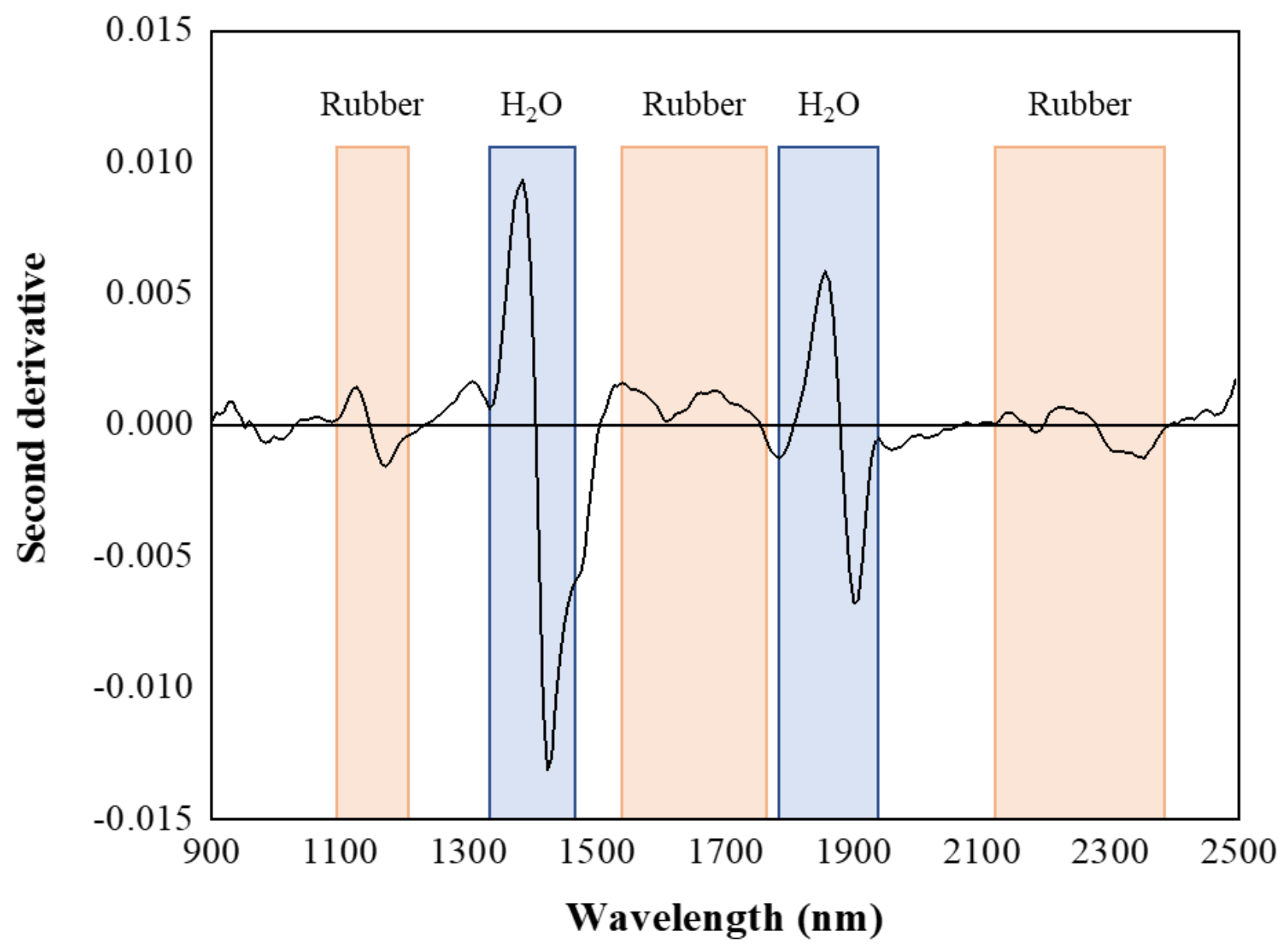

Figure 5

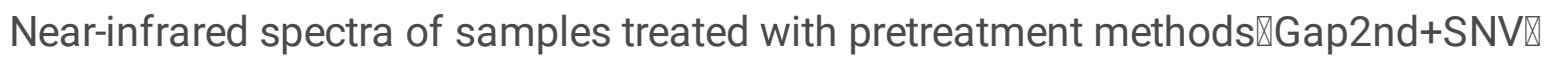

